\title{
ESTIMATIVAS DA RADIAÇÃO DIFUSA DIÁRIA COM BASE NA TRANSMISSIVIDADE ATMOSFÉRICA E INSOLAÇÃO NA TRANSIÇÃO CERRADO-AMAZÔNIA
}

\author{
ZAMADEI, Tamara - tamarazamadei@gmail.com \\ Universidade Federal de Mato Grosso / UFMT
}

\author{
SOUZA, Adilson Pacheco de - adilsonpacheco@ufmt.br \\ Universidade Federal do Mato Grosso / UFMT
ESCOBEDO, João Francisco - escobedo@fca.unesp.br Universidade Estadual Paulista / UNESP \\ ALMEIDA, Frederico Terra de - fredterr@gmail.com \\ Universidade de Federal de Mato Grosso / UFMT
}

\begin{abstract}
RESUMO: Neste estudo avaliaram-se equações de estimativa diárias da radiação solar difusa para o município de Sinop-MT (latitude $11,865^{\circ} \mathrm{S}$, longitude $55,485^{\circ} \mathrm{W}$ e altitude $371 \mathrm{~m}$ ), por meio de correlações das frações radiométricas $\mathrm{Kd}$ e $\mathrm{Kd}^{\prime}$ com o coeficiente de transmissividade atmosférica $\left(K_{T}\right)$ e com a razão de insolação $(n / N)$. Foram geradas e validadas equações estatísticas com agrupamentos anuais, sazonais e mensais. A base de dados empregada na geração das equações foi de junho/2011 a dezembro/2013, enquanto que nas validações foi de janeiro a dezembro de 2014. Os modelos baseados nas correlações entre $\mathrm{Kd}$ e $\mathrm{K}_{\mathrm{T}}$ apresentaram bom desempenho, independentemente do agrupamento de dados. As correlações das frações radiométricas difusas com a razão de insolação propiciam boas estimativas apenas no período chuvoso da região (outubro a abril). As estimativas de $\mathrm{Kd}^{\prime}$ apresentam piores desempenhos estatísticos quando comparados com $\mathrm{Kd}$. Independentemente das frações radiométricas, os modelos geraram subestimativas e superestimativas da radiação difusa nas estações chuvosa e seca, respectivamente. Para aplicações ao longo do ano, recomendam-se as estimativas da radiação difusa com base em medidas da radiação global, com quatro modelos sazonais (estações do ano)..
\end{abstract}

PALAVRAS-CHAVE: índice de claridade, razão de insolação, frações radiométricas.

ESTIMATION OF DAILY DIFFUSE RADIATION BASED ON ATMOSPHERIC TRANSMISSIVITY AND INSOLATION RATIO IN THE CERRADO-AMAZON TRANSITION

ABSTRACT: In this study, equations for estimating the daily diffuse radiation in the Sinop-MT municipality (latitude $11.865^{\circ} \mathrm{S}$, longitude $55.485^{\circ} \mathrm{W}$, and altitude $371 \mathrm{~m}$ ) were evaluated using the correlations of the radiometric fractions, $\mathrm{Kd}$ and $\mathrm{Kd}$, with the atmospheric transmissivity coefficient $\left(\mathrm{K}_{\mathrm{T}}\right)$ and insolation ratio $(\mathrm{n} / \mathrm{N})$. Statistical equations were generated and validated against annual, seasonal, and monthly database groupings. The database used to generate the equations was pertaining to the period from June 2011 to December 2013, while the validations were for January to December 2014. The models based on the correlation between $\mathrm{Kd}$ and $\mathrm{K}_{\mathrm{T}}$ exhibited good performance independently of the database grouping. The models based on the correlation of the diffuse radiometric fractions with the insolation ratio provided good estimates only during the rainfall period (October to April). According to the estimation results, Kd' showed worse statistical performance than $\mathrm{Kd}$. Independently of the radiometric fractions, the statistical models underestimated and overestimated the diffuse radiation during the rainy and dry seasons, respectively. For application annually, estimation of diffuse radiation based on global radiation measurements, with four seasonal models, is recommended. 
KEYWORDS: clearness index, insolation ratio, radiometric fractions.

ESTIMATIVAS DE LA RADIACIÓN DIFUSA DIARIA EN BASE A LA TRANSMISIVIDAD ATMOSFÉRICA E INSOLACIÓN EN LA TRANSICIÓN CERRADO-AMAZONIA

RESUMEN: En este estudio se evaluaron ecuaciones de estimativas diarias de la radiación solar difusa para el municipio de Sinop-MT (latitud $11,865^{\circ} \mathrm{S}$, longitud $55,485^{\circ}$ W y altitud $371 \mathrm{~m}$ ), por medio de correlaciones de las fracciones radiométricas $\mathrm{Kd}$ y $\mathrm{Kd}^{\prime}$ con el coeficiente de transmisividad atmosférica $\left(\mathrm{K}_{\mathrm{T}}\right)$ y con el ratio de insolación $(\mathrm{n} / \mathrm{N})$. Fueron generadas y validadas ecuaciones estadísticas con agrupamientos anuales, estacionales y mensuales. La base de datos empleada en la generación de las ecuaciones fue de junio/2011 a diciembre/2013, mientras que en las validaciones fue de enero a diciembre de 2014. Los modelos basados en las correlaciones entre $\mathrm{Kd}$ y $\mathrm{K}_{\mathrm{T}}$ presentaron un buen rendimiento, independientemente de la agrupación de datos. Las correlaciones de las fracciones radiométricas difusas con el ratio de insolación propician buenas estimativas apenas en el periodo lluvioso de la región (octubre a abril). Las estimativas de $\mathrm{Kd}^{\prime}$ presentan peores rendimientos estadísticos en comparación con $\mathrm{Kd}$. Independientemente de las fracciones radiométricas, los modelos generaron infraestimativas y sobreestimativas de la radiación difusa en las estaciones lluviosa y seca, respectivamente. Para aplicaciones a lo largo del año, se recomiendan las estimativas de la radiación difusa en base a medidas de la radiación global, con cuatro modelos estacionales (estaciones del año).

PALABRAS CLAVE: índice de claridad, ratio de insolación, fracciones radiométricas.

ESTIMATIONS DU RAYONNEMENT DIFFUS JOURNALIER EN FONCTION DE TRANSFERT ATMOSPHÉRIQUE ET INSOLATION EN PASSAGE DE LA SAVANE BRÉSILIEN-AMAZONE

RESUME: Dans cette étude a évalué équations $d^{\prime}$ estimation journalier du rayonnement solaire diffus pour la ville de Sinop-MT (latitude $11,865^{\circ} \mathrm{S}$, longitude $55,485^{\circ} \mathrm{W}$ e altitude $371 \mathrm{~m}$ ), au moyen de les fractions radiométriques $\mathrm{Kd}$ et $\mathrm{Kd}^{\prime}$ avec le coefficient de transfert atmosphérique $\left(\mathrm{K}_{\mathrm{T}}\right)$ et avec le taux de insolation $(\mathrm{n} / \mathrm{N})$. Il y a été génerée et validées les équations statistiques avec groupement annuels, saisonnières et mensuels. La base de données employée à la génération d'équations a été juin/2017 a décembre/2013, alors que aux validations a été janvier a décembre 2014. Les modèles sont basés aux corrélations parmi $\mathrm{Kd}$ et $\mathrm{K}_{\mathrm{T}}$ présentent bonne performance, indépendant du regroupement de données. Les corrélations de la fraction radiométriques diffuses avec la raison d'insolation obtiennent bonnes estimations seulement à saison des pluies de la région (octobre et avril). Les estimations de $\mathrm{Kd}^{\prime}$ présentent le pire résultats, quand comparés avec $\mathrm{Kd}$. Indépendamment de las fraction radiométriques, les modéles ont géneré sous-estimation et surestimation du rayonnement diffus pendant les saisons des pluies et sécheresse, respectivement. Pour applications au fil d'an, il est recommandé les estimations du rayonnement diffus par mesures du rayonnement glonal, avec quatre modèles saisonniers (les saisons d'année).

MOTS CLE: taux de clarté, raison d'insolation, fraction radiométriques.

\section{INTRODUÇÃO}

Os níveis da radiação solar incidente na superfície terrestre são informações fundamentais para aplicações voltadas à energia solar, agricultura, arquitetura, climatologia, iluminação, sistemas de conversão energética, dentre outras (BASHAHU, 2003; KUMAR e UMANAND, 2005). Em planos horizontais, a radiação global é composta pelas componentes direta e difusa. As estações meteorológicas brasileiras monitoram apenas a radiação global em função dos custos de aquisição e operação dos sensores, justificando, assim, o uso de modelos que estimem as demais componentes da radiação solar. 
Na estimativa da radiação solar normalmente são empregados dois tipos de modelo: estatísticos (ou de decomposição) e parametrizados. Os modelos estatísticos são representados por equações matemáticas ajustadas por meio de regressões em correlações com a razão de insolação e/ou com as frações radiométricas. Nesse caso, o emprego de frações das diferentes componentes da radiação solar, permite a minimização dos efeitos geográficos e astronômicos, visto que se consideram componentes obtidas no mesmo local, instante e intervalo de tempo. Já os modelos parametrizados carecem de informações mais detalhadas das condições atmosféricas locais, conforme a componente a ser estimada, ou então a utilização de percentuais fixos de ocorrência de cada componente da radiação solar global.

Liu e Jordan (1960) foram os precursores da utilização da correlação entre a fração difusa $(\mathrm{Kd})$ e o coeficiente de transmissividade atmosférica $\left(\mathrm{K}_{\mathrm{T}}\right)$, que indicam as razões da radiação difusa com a radiação global e da radiação global com a radiação no topo da atmosfera, respectivamente. Posteriormente, muitos trabalhos foram desenvolvidos para calibração e alteração desse modelo com diferentes dados, regiões e escalas temporais (ORGILL e HOLLANDS, 1977; IQBAL, 1979; BARBARO et al., 1981; JAIN, 1990; REINDLE et al., 1990; TASDEMIROGLU e SEVER, 1991; OLIVEIRA et al., 2002; JACOVIDES et al., 2006; MUNEER e MUNAWWAR, 2006; ELMINIR, 2007; ELMINIR et al., 2007; JIANG, 2009; ULGEN e HEPBASLI, 2009; BUTT et al., 2010; LI et al., 2011; BOUKELIA et al., 2014; KUO et al., 2014).

Tais correlações apresentam grande variabilidade em função da partição temporal escolhida, do local estudado e do tipo de ajuste. Em geral, são obtidos ajustes lineares, polinomiais (primeira a quarta ordem) e exponenciais, no entanto, a escolha dependerá do desempenho estatístico dos modelos gerados localmente (DAL PAI e ESCOBEDO, 1999; DERVISHI e MAHDAVI, 2012). Segundo Muneer e Munawwar (2006), o emprego de parâmetros meteorológicos como a razão de insolação e/ou fração de nuvens presentes na atmosfera podem melhorar o desempenho de modelos de estimativa da radiação difusa.

Além disso, é fundamental a avaliação do desempenho dos modelos estatísticos gerados com outras variáveis meteorológicas ou componentes da radiação solar, com a indicação dos desvios médios, ocorrências de super ou subestimativas, espalhamentos e ajustamentos entre valores estimados e observados (SOUZA ET AL., 2011; BERUSKI ET AL., 2015; TEKE ET AL., 2015).

Neste trabalho objetivou-se gerar e validar equações de estimativa da radiação difusa com base no coeficiente de transmissividade atmosférica e na razão de insolação, na partição diária e em diferentes agrupamentos, na região de transição Cerrado-Amazônia - Sinop-MT.

\section{MATERIAL E MÉTODOS}

Os valores das irradiâncias global e difusa medidas na superfície horizontal foram obtidos da base de dados da Estação Meteorológica Automática da UFMT Campus Universitário de Sinop (latitude $11,865^{\circ} \mathrm{S}$, longitude $55,485^{\circ}$ W e altitude $371 \mathrm{~m}$ ), dentre o período de 02 de junho de 2011 a 31 de dezembro de 2014. 
O clima da região segundo a classificação climática de Köppen é do tipo Aw tropical quente e úmido, caracterizado pela presença de duas estações bem definidas: chuvosa (outubro a abril) e seca (maio a setembro). As temperaturas médias mensais oscilam entre $23^{\circ} \mathrm{C}$ e $25,8^{\circ} \mathrm{C}$, com valor médio anual de $24,7^{\circ} \mathrm{C}$. Em relação à precipitação ocorrem em média $1900 \mathrm{~mm}$ ano-1 $^{-1}$, com concentrações de $70 \%$ do total de chuvas entre novembro e março (verão), sendo que os meses mais chuvosos estão no intervalo de janeiro a março. Durante o primeiro trimestre do ano a precipitação chega a atingir de $45 \%$ a $55 \%$ do total anual das chuvas (SETTE e TARIFA, 2000; SOUZA et al., 2013; ALVARES et al., 2014).

As médias mensais da temperatura do ar, umidade relativa, precipitação e insolação observadas no local de estudo, durante o período de tempo em questão, são apresentadas na Figura 1.

A

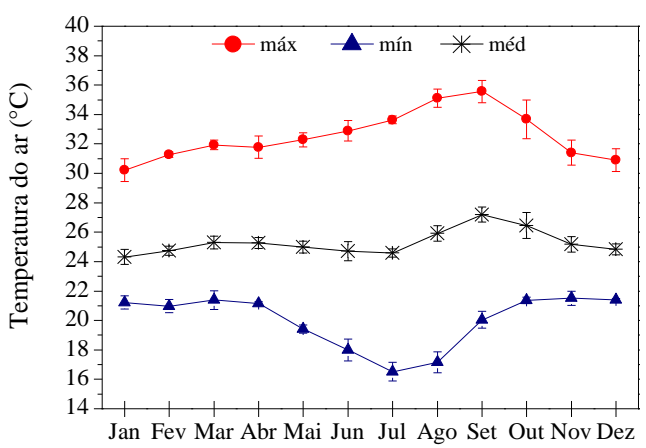

C.

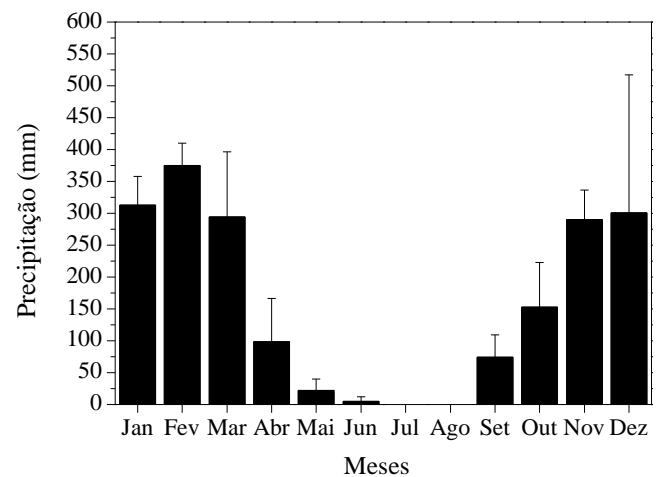

B.

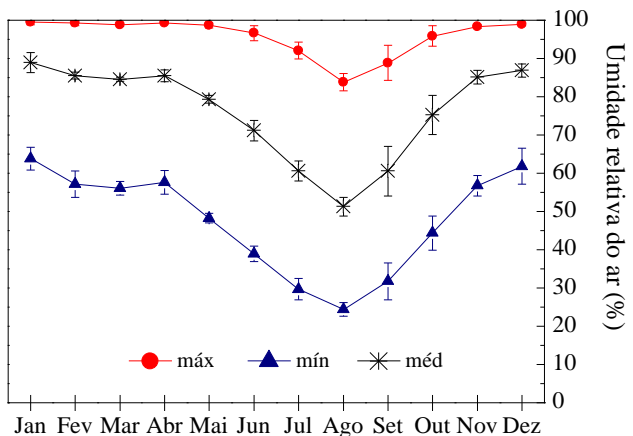

D.

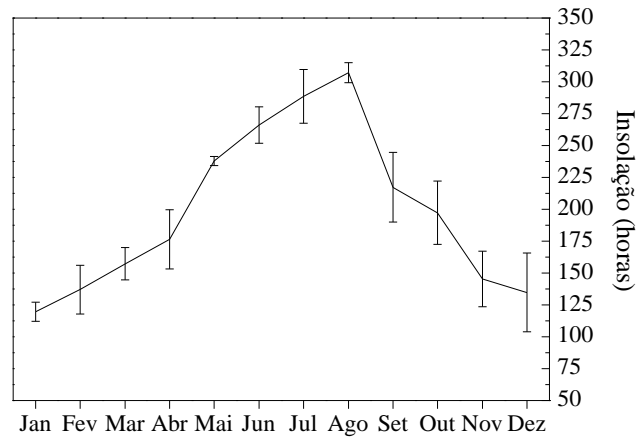

Meses

Figura 1 - Evolução média anual das variáveis meteorológicas: temperatura $(A)$, umidade relativa do ar (B), precipitação acumulada (C), insolação total (D), para a região de Sinop-MT, entre 02/06/2011 a 31/12/2014

As irradiâncias global (IG) e difusa (IDF) foram monitoradas por piranômetros CM3 da Kipp \& Zonen, posicionados a $1 \mathrm{~m}$ de altura, que possuem uma sensibilidade de resposta de $\pm 10-35 \mu \mathrm{V} / \mathrm{Wm}^{-2}$, tempo de resposta de $18 \mathrm{~s}$, resposta a temperatura de $\pm 1,0 \%$ para a faixa de -40 a $80{ }^{\circ} \mathrm{C}$ e desvios para 0 efeito cosseno de $\pm 2 \%\left(0<z<80^{\circ}\right)$; sendo a difusa medida com base no anel de sombreamento MEO (MELO, 1994). Na aquisição dos dados foi utilizado um datalogger CR1000 (Campbell Scientific) operando na frequência de $1 \mathrm{~Hz}$ e armazenando médias de 300 leituras ou 5 min. 
Os dados de IG e IDF sofreram análise para observação de inconsistências geradas pelo sistema de coleta e armazenamento de dados. Com base em integrações das partições instantâneas foram obtidas as irradiações diárias extraterrestre $\left(H_{0}\right)$, global $\left(H_{G}\right)$ e difusa $\left(H_{D F}\right)$ (IQBAL, 1983). Os dados de $\mathrm{H}_{\mathrm{DF}}$ foram submetidos a correções geométricas e anisotrópicas através das aplicações de fatores de correção propostos por Oliveira et al. (2002) e Dal Pai et al. (2011). Posteriormente, realizou-se a filtragem da base de dados diária, considerando-se os critérios descritos por Escobedo et al. (2009), sendo: i) $H_{G} /$ $\mathrm{H}_{0}<0,82$; e ii) $\mathrm{H}_{D F} / \mathrm{H}_{\mathrm{G}}<1,0$.

O coeficiente de transmissividade atmosférica da radiação global $\left(K_{T}\right)$ ou índice de claridade foi obtido pela razão entre a radiação global $\left(\mathrm{H}_{\mathrm{G}}\right)$ e a radiação no topo da atmosfera $\left(\mathrm{H}_{0}\right)$. Posteriormente, estabeleceu-se a classificação da cobertura de céu em função de Kt proposta por Escobedo et al. (2009), a qual dispensa o uso de dados de radiação direta e/ou difusa, e considera os tipos de condição de céu expostos na Tabela 1 . Em seguida, realizou-se a distribuição de frequência da base de dados por condição de cobertura de céu.

Tabela 1 - Classificação da cobertura de céu em função de $K_{T}$ proposta por Escobedo et al. (2009)

\begin{tabular}{cc}
\hline Intervalo de $\mathrm{K}_{\mathrm{T}}$ & Cobertura de céu \\
\hline $0 \leq \mathrm{K}_{\mathrm{T}}<0,35$ & Nublado \\
$0,35 \leq \mathrm{K}_{\mathrm{T}}<0,55$ & Parcialmente nublado \\
$0,55 \leq \mathrm{K}_{\mathrm{T}}<0,65$ & Parcialmente aberto \\
$\mathrm{K}_{\mathrm{T}} \geq 0,65$ & Aberto \\
\hline
\end{tabular}

As frações radiométricas $\mathrm{Kd}$ e $\mathrm{Kd}^{\prime}$ representam a parcela da radiação global incidente na superfície terrestre como radiação difusa, calculadas pela razão entre a radiação difusa e a radiação global, e a difusa pela radiação no topo da atmosfera, respectivamente.

Foram avaliadas as correlações entre as frações radiométricas $\mathrm{Kd}$ e $\mathrm{Kd}^{\prime}$ com o coeficiente de transmissividade global diário - $\mathrm{K}_{\mathrm{T}}$ e a razão de insolação $\mathrm{n} / \mathrm{N}$ (calculada com base na duração do brilho solar observado e duração astronômica do dia.) Foram geradas equações estatísticas para agrupamentos de dados mensais, sazonais (estação do ano) e anuais, com suas respectivas validações, e ainda, com validações cruzadas entre os agrupamentos. Na geração das equações foram empregados os dados de junho de 2011 a dezembro de 2013, enquanto que na validação, foram utilizados os dados de 2014.

Foram avaliados diferentes modelos estatísticos para as correlações geradas, todavia, os melhores ajustes foram dados por polinômios de segunda ordem, corroborando com a literatura (BASHAHU, 2003; DAL PAI, 2005; JIANG, 2009; KOUSSA et al., 2009; SOUZA e ESCOBEDO, 2013).

Para avaliação do grau de desempenho estatístico foram empregados diferentes indicativos estatísticos: i) coeficientes de determinação $\left(R^{2}\right)$; ii) coeficiente de Pearson, conforme metodologia proposta por Devore (2006) 
(Tabela 2); iii) MBE (desvio das médias) (Eq. 1); iv) RMSE (erro médio quadrático) (Eq. 2); v) índice de ajustamento (d) de Willmott (1981) (Eq. 3). A utilização conjunta de diferentes indicadores estatísticos, pode ser considerada como alternativa para validação de modelos estatísticos, pois possibilita a análise simultânea dos desvios dos valores médios, ocorrência de sub ou superestimativa, espalhamento e ajustamento entre valores estimados e medidos (SOUZA et al., 2011; BERUSLKI et al., 2015; TEKE et al., 2015; TANAKA et al., 2016).

$O$ indicativo MBE representa o desvio das médias e provê informações quanto à performance do modelo a longo prazo. Valores negativos de MBE indicam subestimativa do modelo testado, e vice-versa. Segundo Stone (1993), quanto menor o valor absoluto de $\mathrm{MBE}$, melhor é a performance do modelo testado. A desvantagem deste método é que uma superestimativa cancela uma subestimativa. O RMSE é a raiz quadrada do erro quadrático médio e informa sobre o valor real do erro produzido pelo modelo. As desvantagens do RMSE são que alguns erros de grande proporção na soma podem causar acréscimos significativos nos valores de RMSE, além do que ele não diferencia superestimativa de subestimativa. Em geral, quanto menores os valores obtidos para RMSE, melhor performance dos modelos. O índice de ajustamento " $\mathrm{d}$ " varia de 0 a 1 e representa o quanto os valores estimados se ajustam com os valores medidos (WILLMOTT, 1981).

Tabela 2 - Grau da correlação pelo coeficiente de Pearson ( $r$ ) proposto por Devore (2006)

\begin{tabular}{cc}
\hline Coeficiente de Pearson $(r)$ & Definição \\
\hline $0,00-0,19$ & Correlação bem fraca \\
$0,20-0,39$ & Correlação fraca \\
$0,40-0,69$ & Correlação moderada \\
$0,70-0,89$ & Correlação forte \\
$0,90-1,00$ & Correlação muito forte \\
\hline
\end{tabular}

$M B E=\frac{\sum_{i=1}^{N}(P i-O i)}{N}$

$\operatorname{RMSE}=\left[\frac{\sum_{\mathrm{i}=1}^{\mathrm{N}}(\mathrm{Pi}-\mathrm{Oi})^{2}}{\mathrm{~N}}\right]^{\frac{1}{2}}$

$d=1-\frac{\sum_{i=1}^{N}(P i-O i)^{2}}{\sum_{i=1}^{N}\left(\left|P^{\prime} i\right|+\left|O^{\prime} i\right|\right)^{2}}$ 
em que: $\mathrm{Pi}$ - valores estimados; Oi - valores medidos; $\mathrm{N}$ - número de observações; $\left|\mathrm{P}^{\prime} \mathrm{i}\right|$ - valor absoluto da diferença $P i-\overline{O i} ;\left|\mathrm{O}^{\prime} \mathrm{i}\right|$ - valor absoluto da diferença $\mathrm{Oi}-\overline{\mathrm{Oi}}$.

Considerando os indicativos estatísticos supracitados e visando analisar/concluir quanto ao desempenho dos modelos gerados, empregou-se o método dos valores de posição ( $V p)$. Segundo Tanaka et al. (2016), nesse caso, atribuem-se pesos de 1 a $\mathrm{n}$ para cada indicador estatístico, em que $\mathrm{n}$ é 0 número de modelos testados que, nesse caso, tiveram peso 1 , para o modelo com melhor desempenho, e peso $n$, para o modelo com pior desempenho. A indicação dos modelos de acordo com seu desempenho foi realizada pelo somatório dos pesos atribuídos, ou seja, o menor Vp acumulado indica o melhor modelo.

\section{RESULTADOS E DISCUSSÃO}

A variação diária das radiações global e difusa que atinge a superfície horizontal, obtida pela integração da partição horária, pode ser observada na Figura 2. Os valores médios diários observados para a radiação global e difusa foram de 19,26 e 5,98 $\mathrm{MJ} \mathrm{m}^{-2} \mathrm{~d}^{-1}$, respectivamente. Os maiores valores da radiação difusa podem ser observados durante o período de outubro a abril, correspondente à estação chuvosa na região estudada, devido à maior concentração de nuvens. Os valores mais altos são verificados no mês de janeiro, enquanto os menores são observados em julho.

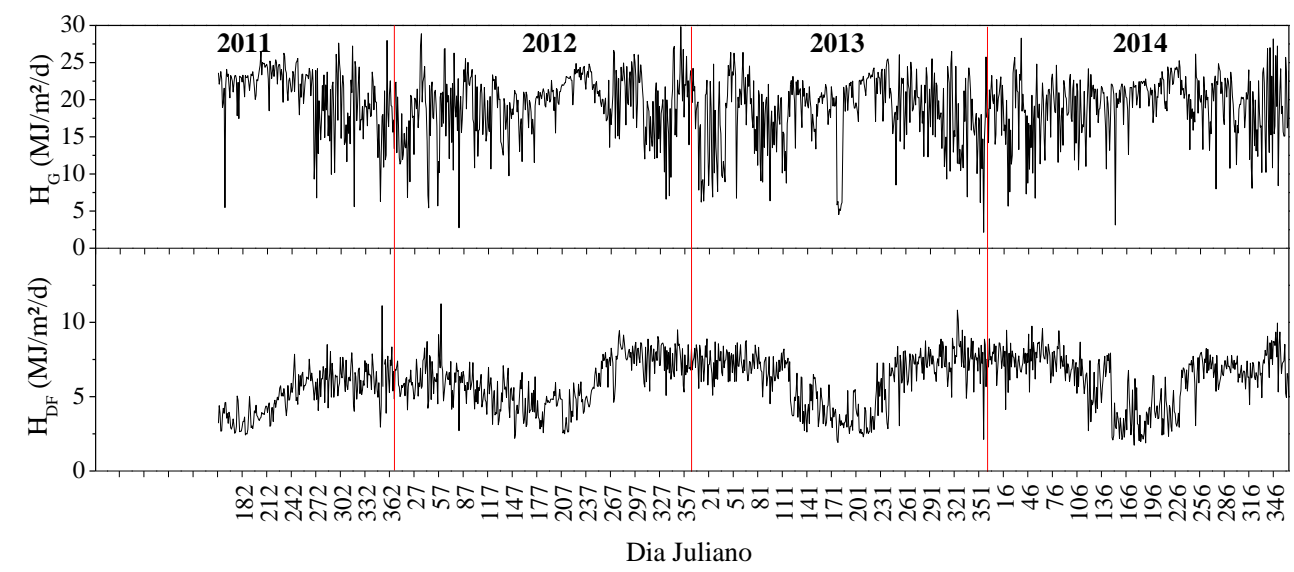

Figura 2 - Valores diários da radiação solar global e difusa (MJ $\left.\mathrm{m}^{-2} \mathrm{~d}^{-1}\right)$ na região de Sinop-MT, entre 02/06/2011 a 31/12/2014

A Tabela 3 apresenta as frequências das condições de céu em Sinop durante o período de estudo. Na Figura 3 pode-se observar as correlações propostas entre as frações radiométricas e $K_{T}$ e $n / N$ para o agrupamento de dados anual. Segundo Souza et al. (2016) o comportamento das condições de cobertura de céu ao longo do ano na região amazônica de Mato Grosso é dependente do comportamento pluviométrico regional e das modificações na 
composição da atmosfera. Notadamente observa-se que entre maio e agosto, acima de $90 \%$ dos dias apresentaram transmissividade atmosférica da radiação global superior a $55 \%$ (classes de céu parcialmente aberto e aberto). Em contrapartida, com elevação dos valores de $\mathrm{K}_{\mathrm{T}}$, espera-se menores variações da razão de insolação, e consequentemente, maiores limitações em estimativas que empregam $\mathrm{n} / \mathrm{N}$ como variável independente.

Na Figura $3 \mathrm{~A}$, nos limites (céu nublado e céu aberto), quando $\mathrm{K}_{\mathrm{T}}$ tende a zero, $\mathrm{Kd}$ tende a um, e quando $\mathrm{Kd}$ tende a zero, $\mathrm{K}_{\mathrm{T}}$ tende a 0,82 . No intervalo intermediário, $\mathrm{K}_{\mathrm{T}}$ pode assumir tendência linear, polinomial, de $2^{\mathrm{a}}, 3^{\mathrm{a}}$ ou $4^{\mathrm{a}}$ ordens, ou mesmo uma exponencial (RECIERI et al., 2002). Para a variação da razão de insolação e consequentemente da cobertura de céu, ocorre um valor de transmissividade máxima para a radiação difusa que, no entanto, não ocorre em uma condição de céu completamente nublado $(\mathrm{n} / \mathrm{N}=0)$. Este comportamento polinomial decorre das variações da presença de vapor d'água e/ou partículas em suspensão, que causam espalhamento e absorção ao mesmo tempo, e em conjunto, influenciam diretamente na sazonalidade da radiação incidente na superfície (SOUZA e ESCOBEDO, 2013).

Tabela 3 - Frequências das condições de céu em Sinop -MT ao longo do ano de 2014 de acordo com classificação proposta por Escobedo et al. (2009)

\begin{tabular}{ccccc}
\hline & \multicolumn{4}{c}{ Condição de céu } \\
\cline { 2 - 5 } Mês & I & II & III & IV \\
& (nublado) & $\begin{array}{c}\text { (parcialmente } \\
\text { nublado) }\end{array}$ & (parcialmente aberto) & (aberto) \\
\hline Janeiro & 22,58 & 58,06 & 19,35 & 0,00 \\
Fevereiro & 25,00 & 57,14 & 14,29 & 3,57 \\
Março & 9,68 & 61,29 & 25,81 & 3,23 \\
Abril & 10,00 & 40,00 & 33,33 & 16,67 \\
Maio & 0,00 & 9,68 & 35,48 & 54,84 \\
Junho & 3,33 & 3,33 & 6,67 & 86,67 \\
Julho & 0,00 & 0,00 & 9,68 & 90,32 \\
Agosto & 0,00 & 3,23 & 38,71 & 58,06 \\
Setembro & 0,00 & 56,67 & 36,67 & 6,67 \\
Outubro & 9,68 & 45,16 & 41,94 & 3,23 \\
Novembro & 23,33 & 60,00 & 16,67 & 0,00 \\
Dezembro & 12,90 & 48,39 & 25,81 & 12,90 \\
\hline
\end{tabular}




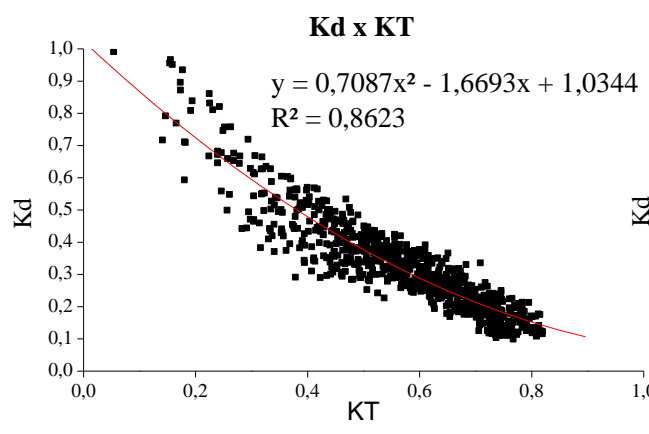

Kd' x KT

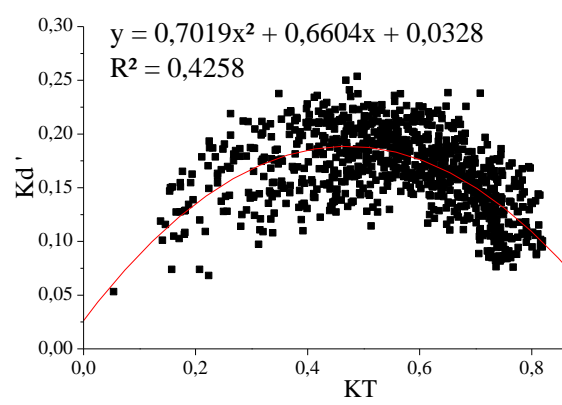

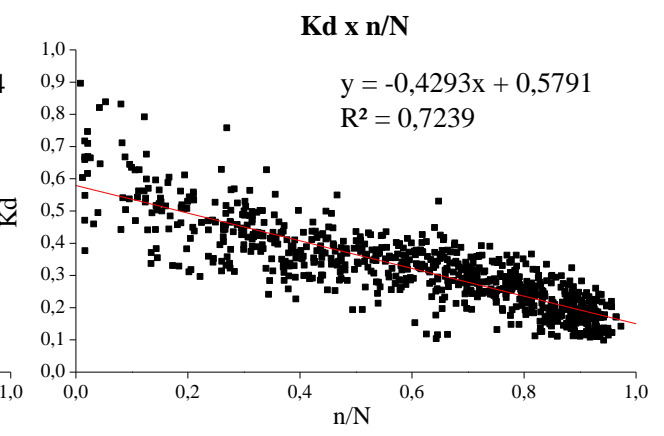

$\mathbf{K d} \mathbf{d}^{\prime} \mathbf{x} \mathbf{n} / \mathbf{N}$

Figura 3 - Correlações entre $K d$ e $K T, K d$ e $n / N, K d^{\prime}$ e $K T$ e $K d^{\prime}$ e $n / N$ para $o$ agrupamento anual de dados, estabelecidas com os valores observados dentre 02/06/2011 e 31/12/2013 na região de Sinop-MT.

A fração $\mathrm{Kd}$ apresentou melhores estimativas com base no coeficiente de transmissividade atmosférica quando comparado com a razão de insolação (Tabela 4). Os modelos $\mathrm{Kd} \times \mathrm{K}_{\mathrm{T}}$ apresentaram bons ajustes em praticamente todos os períodos, com $\mathrm{R}^{2}$ acima de 0,7; exceto em julho e agosto (inverno). Tais meses do período seco apresentam maiores frequências da cobertura de céu tipo IV (Tabela 3) conforme classificação proposta por Escobedo et al. (2009), que indica que $\mathrm{K}_{\mathrm{T}} \geq 0,65$, e portanto tem-se grande intervalo de pontos da variável dependente $(\mathrm{Kd})$ para um pequeno intervalo de $\mathrm{K}_{\mathrm{T}}(0,65$ a 0,82$)$, 0 que resulta em um ajuste de desempenho inferior da linha de tendência aos dados.

Os meses de maio e junho, também da epoca de seca, apesar de apresentarem alta frequência de céu aberto $(54,84$ e 86,67\%, respectivamente), possuem maior número de valores dentro das classes céu nublado (I) e parcialmente difuso (II) em comparação a julho e agosto, o que melhora o desempenho do ajuste. De acordo com o coeficiente de correlação (r) e a classificação proposta por Devore (2006), as correlações observadas são fortes, com r variando de 0,77 a 0,94.

A correlação $\mathrm{Kd} \times \mathrm{n} / \mathrm{N}$ mostrou-se mais fraca quando comparada com $\mathrm{K}_{\mathrm{T}}$, com valores mínimos e máximos de $R^{2}$ iguais a 0,30 e 0,72. O comportamento dos meses de julho e agosto segue o mesmo padrão nesta correlação. Apesar do baixo desempenho dos modelos mensais e sazonais, a equação anual apresentou ótimo desempenho, com $R^{2}=0,72(r=0,85)$.

Dal Pai (2005) afirma que a componente difusa apresenta comportamento anisotrópico e isotrópico e, normalmente, é composta pelas 
subcomponentes circunsolar, brilho horizontal (multirreflexões) e radiação difusa isotrópica e que, em muitos modelos de estimativa, não são contempladas, como no caso da correlação com a razão de insolação.

Tabela 4 - Coeficientes de regressão das equações de estimativa da radiação solar difusa na equipartição diária para a região de Sinop-MT, geradas através da correlação de $\mathrm{Kd}$ com $\mathrm{K}_{\mathrm{T}}$ e $\mathrm{n} / \mathrm{N}$

\begin{tabular}{cccccccc}
\hline & \multicolumn{4}{c}{$\mathrm{Kd} \times \mathrm{K}_{\mathrm{T}}$} & \multicolumn{2}{c}{$\mathrm{Kd} \times \mathrm{n} / \mathrm{N}$} \\
\hline Período & $\mathrm{a}_{0}$ & $\mathrm{a}_{1}$ & $\mathrm{a}_{2}$ & $\mathrm{R}^{2}$ & $\mathrm{a}_{0}$ & $\mathrm{a}_{1}$ & $\mathrm{R}^{2}$ \\
\hline Janeiro & 1,5379 & $-4,8944$ & 4,9857 & $0,7085^{*}$ & 0,6399 & $-0,6087$ & $0,5035^{* *}$ \\
Fevereiro & 1,1622 & $-2,2629$ & 1,3488 & $0,8753^{*}$ & 0,6476 & $-0,5158$ & $0,6535^{*}$ \\
Março & 1,1218 & $-2,1053$ & 1,2038 & $0,8620^{*}$ & 0,6233 & $-0,4996$ & $0,6702^{*}$ \\
Abril & 1,1673 & $-2,1783$ & 1,229 & $0,7769^{*}$ & 0,6088 & $-0,4672$ & $0,6737^{*}$ \\
Maio & 0,3917 & 0,6883 & $-1,3812$ & $0,7600^{*}$ & 0,5328 & $-0,3920$ & $0,5464^{*}$ \\
Junho & 1,1808 & $-2,0871$ & 0,9913 & $0,8716^{*}$ & 0,6223 & $-0,5018$ & $0,6301^{*}$ \\
Julho & 1,6429 & $-3,3424$ & 1,8349 & $0,5878^{* *}$ & 0,5096 & $-0,3740$ & $0,3016^{\text {ns }}$ \\
Agosto & 1,6777 & $-3,4293$ & 1,8983 & $0,6342^{* *}$ & 0,5981 & $-0,4635$ & $0,3715^{* *}$ \\
Setembro & 0,9727 & $-1,3893$ & 0,4506 & $0,8063^{*}$ & 0,5826 & $-0,3992$ & $0,6393^{* *}$ \\
Outubro & 0,7652 & $-0,8239$ & 0,148 & $0,7258^{*}$ & 0,5135 & $-0,2704$ & $0,5829^{* *}$ \\
Novembro & 0,9233 & $-1,3452$ & 0,5011 & $0,7361^{*}$ & 0,5605 & $-0,3732$ & $0,4529^{* *}$ \\
Dezembro & 1,2226 & $-2,5468$ & 1,678 & $0,8716^{*}$ & 0,5829 & $-0,4467$ & $0,5931^{*}$ \\
\hline Verão & 1,1831 & $-2,5021$ & 1,6965 & $0,8222^{*}$ & 0,5621 & $-0,3923$ & $0,5079^{* *}$ \\
Outono & 1,0907 & $-1,7866$ & 0,7547 & $0,8729^{*}$ & 0,5914 & $-0,4588$ & $0,6924^{*}$ \\
Inverno & 1,1095 & $-1,8056$ & 0,7442 & $0,8196^{*}$ & 0,4838 & $-0,3260$ & $0,4397^{* *}$ \\
Primavera & 1,0706 & $-1,9955$ & 1,2098 & $0,7747^{*}$ & 0,5498 & $-0,3447$ & $0,5921^{*}$ \\
\hline Anual & 1,0344 & $-1,6693$ & 0,7087 & $0,8623^{*}$ & 0,5791 & $-0,4293$ & $0,7239^{*}$ \\
\hline
\end{tabular}

ns = não significativo; ${ }^{*}$ e ${ }^{* *}=$ significativos a 1 e $5 \%$ de probabilidade, respectivamente.

A fração $\mathrm{Kd}^{\prime}$ apresentou baixa correlação (não significância a $5 \%$ de probabilidade) com $\mathrm{K}_{\mathrm{T}}$ e $\mathrm{n} / \mathrm{N}$ (Tabela 5 ), uma vez que há grande influência da composição atmosférica. O coeficiente de determinação varia de 0,02 a 0,48, sendo consideradas como correlações de fracas a moderadas (DEVORE, 2006). Pelo comportamento supracitado para a correlação $\mathrm{Kd}^{\prime} \times \mathrm{K}_{\mathrm{T}}$, as curvas apresentam crescimento até o ponto máximo em que $K_{T}=0,53$. Dal Pai (2001) cita que os valores máximos ocorrem na cobertura de céu parcialmente nublado, o que causa um aumento do fluxo difuso em virtude do comportamento complexo dessas condições de cobertura do céu, mesclando as componentes difusa e direta da irradiação ao mesmo tempo. 
Tabela 5 - Coeficientes de regressão das equações de estimativa da radiação solar difusa na partição diária para a região de Sinop-MT, geradas através da correlação de Kd' com $\mathrm{K}_{\mathrm{T}}$ e $\mathrm{n} / \mathrm{N}$

\begin{tabular}{ccccccccc}
\hline \multicolumn{9}{c}{$\mathrm{Kd}^{\prime} \times \mathrm{K}_{\mathrm{T}}$} \\
\hline Período & $\mathrm{a} 0$ & $\mathrm{a} 1$ & $\mathrm{a} 2$ & $\mathrm{R}^{2}$ & $\mathrm{a} 0$ & $\mathrm{~K} 1$ & $\mathrm{~K} 2$ & $\mathrm{R}^{2} \times \mathrm{n} / \mathrm{N}$ \\
\hline Janeiro & 0,1549 & $-0,0828$ & 0,2480 & $0,2401^{\text {ns }}$ & 0,1556 & $-0,0053$ & 0,0908 & $0,1309^{\text {ns }}$ \\
Fevereiro & 0,0837 & 0,4404 & $-0,4712$ & $0,2434^{\text {ns }}$ & 0,1701 & 0,0678 & $-0,0799$ & $0,0458^{\text {ns }}$ \\
Março & 0,0419 & 0,6268 & $-0,6663$ & $0,3404^{* *}$ & 0,1586 & 0,139 & $-0,1631$ & $0,1085^{\text {ns }}$ \\
Abril & 0,0790 & 0,5571 & $-0,6335$ & $0,2009^{\text {ns }}$ & 0,1937 & 0,0685 & $-0,1307$ & $0,2284^{\text {ns }}$ \\
Maio & $-0,2185$ & 1,6170 & $-1,5834$ & $0,4400^{* *}$ & 0,1915 & 0,0046 & $-0,0772$ & $0,193^{\text {ns }}$ \\
Junho & 0,0112 & 0,6720 & $-0,6795$ & $0,4421^{* *}$ & 0,1496 & 0,1708 & $-0,2178$ & $0,3347^{\text {ns }}$ \\
Julho & 0,0302 & 0,5239 & $-0,5217$ & $0,2292^{\text {ns }}$ & 0,2117 & $-0,084$ & $-0,0108$ & $0,0821^{\text {ns }}$ \\
Agosto & 0,4616 & $-0,6553$ & 0,2847 & $0,2387^{\text {ns }}$ & $-0,1308$ & 0,8977 & $-0,6738$ & $0,2165^{\text {ns }}$ \\
Setembro & $-0,0407$ & 0,9894 & $-1,0259$ & $0,4289^{* *}$ & 0,1569 & 0,1927 & $-0,2244$ & $0,1811^{\text {ns }}$ \\
Outubro & $-0,0192$ & 0,9318 & $-0,9892$ & $0,3135^{\text {ns }}$ & 0,1629 & 0,0842 & $-0,0628$ & $0,0458^{\text {ns }}$ \\
Novembro & 0,0467 & 0,6346 & $-0,6946$ & $0,1639^{\text {ns }}$ & 0,1629 & 0,1198 & $-0,1325$ & $0,0632^{\text {ns }}$ \\
Dezembro & 0,0544 & 0,4883 & $-0,4628$ & $0,2599^{\text {ns }}$ & 0,1628 & 0,0656 & $-0,068$ & $0,0239^{\text {ns }}$ \\
\hline Verão & 0,0728 & 0,4368 & $-0,4375$ & $0,2295^{\text {ns }}$ & 0,1584 & 0,0919 & $-0,091$ & $0,0694^{\text {ns }}$ \\
Outono & 0,0663 & 0,5873 & $-0,6699$ & $0,4754^{* *}$ & 0,1815 & 0,0995 & $-0,1715$ & $0,3911^{\text {ns }}$ \\
Inverno & $-0,0073$ & 0,7739 & $-0,7854$ & $0,4440^{* *}$ & 0,152 & 0,2008 & $-0,2502$ & $0,3074^{\text {ns }}$ \\
Primavera & 0,0584 & 0,4957 & $-0,4702$ & $0,1552^{\text {ns }}$ & 0,1637 & 0,0928 & $-0,0913$ & $0,0424^{\text {ns }}$ \\
\hline Anual & 0,0328 & 0,6604 & $-0,7019$ & $0,4258^{* *}$ & 0,1537 & 0,1788 & $-0,2218$ & $0,3256^{\text {ns }}$ \\
\hline ns não
\end{tabular}

ns = não significativo; ${ }^{*}$ e $* *=$ significativos a 1 e $5 \%$ de probabilidade, respectivamente.

Os valores médios diários observados e estimados da radiação solar difusa para cada período, de acordo com os modelos ajustados, podem ser observados na Figura 4. Os valores dos indicativos estatísticos das equações geradas a partir das correlações mensais, sazonais e anuais entre $\mathrm{Kd}$ e $\mathrm{Kd}^{\prime} \times \mathrm{K}_{\mathrm{T}}$ e n/N são apresentados nas Tabelas 6 e 7.

Através da análise do indicativo estatístico $\mathrm{MBE}$, que representa o desvio das médias, pode-se afirmar que as correlações apresentaram comportamento de estimativa diferentes, com tendência a subestimar os valores da radiação difusa. Oliveira et al. (2002) constataram comportamento semelhante em estudo da correlação Kd e KT no município de São Paulo-SP. Na correlação Kd x $\mathrm{K}_{\mathrm{T}}$, para as equações mensais, MBE variou de $-0,042$ (julho) a 0,958 (junho), sendo que os menores valores são observados em meses de 2014 que apresentaram menor variação nas precipitações acumuladas quando comparada aos demais anos empregados na geração dos modelos. 


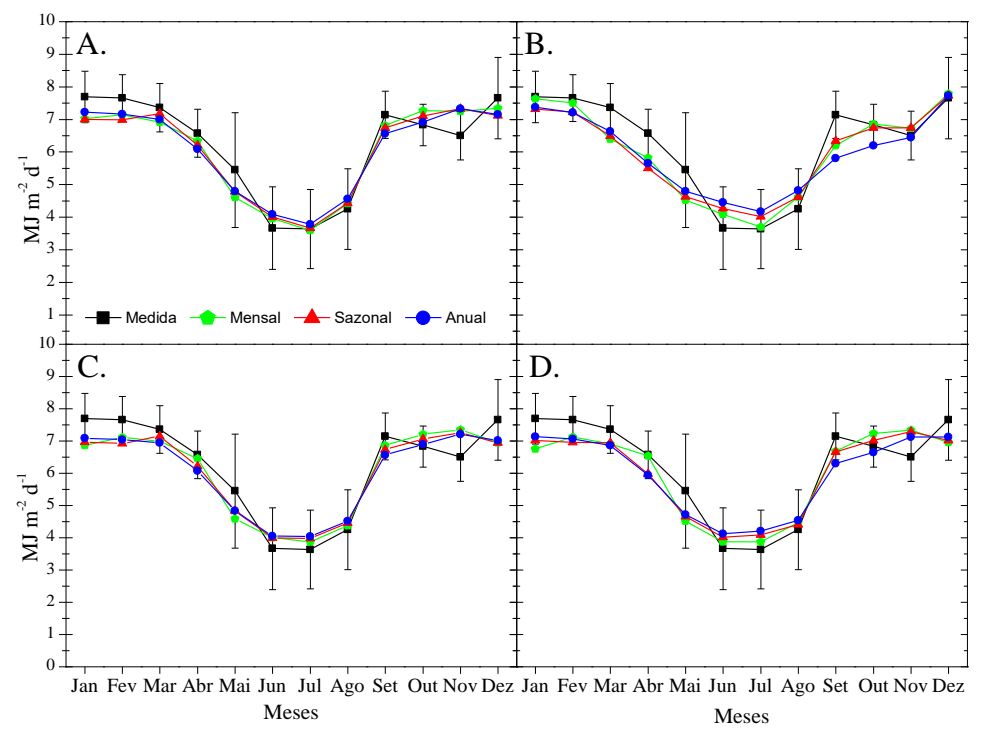

Figura 4 - Valores observados e estimados da radiação difusa para a região de SinopMT, a partir de equações geradas através das correlações $K d \times K_{T}(A), K d \times n / N(B), K d ' x$ $\mathrm{K}_{\mathrm{T}}(\mathrm{C})$ e $\mathrm{Kd} \mathrm{d}^{\prime} \mathrm{x} \mathrm{n} / \mathrm{N}(\mathrm{D})$, para o ano de 2014

Os graus de espalhamento (RMSE) obtidos na correlação $\mathrm{Kd} \times \mathrm{K}_{\mathrm{T}}$ variam de 0,66 a 1,75 $\mathrm{MJ} \mathrm{m}^{-2}$ nas equações mensais; 0,82 a 1,07 $\mathrm{MJ} \mathrm{m}^{-2}$ nas sazonais; e $1,03 \mathrm{MJ} \mathrm{m}^{-2}$ na equação anual. Na correlação $\mathrm{Kd} \times \mathrm{n} / \mathrm{N}$ os valores variam entre 0,70 e $1,83 \mathrm{MJ} \mathrm{m}^{-2}$ nas equações mensais; 0,58 e 1,79 $\mathrm{MJ} \mathrm{m}^{-2}$ nas sazonais; e $1,33 \mathrm{MJ} \mathrm{m}^{-2}$ na anual. Para as correlações estabelecidas com a fração $\mathrm{Kd}^{\prime}$, o RMSE observado para as equações anuais foi de 0,971 ( $\left.\mathrm{K}_{\mathrm{T}}\right)$ e 1,050 (n/N). Os valores se apresentam dentro dos padrões descritos na literatura para essa partição temporal (OLIVEIRA et al., 2002; BASHAHU, 2003; ESCOBEDO et al., 2004; DAL PAI, 2005; ULGEN e HEPBASLI, 2009).

Gomes e Escobedo (2002) afirmam que os valores de radiação difusa são dependentes da concentração variável de moléculas suspensas na atmosfera e de partículas (aerossóis), as quais promovem o espalhamento ou difusão das bandas do espectro da radiação, justificando assim o maior espalhamento dos pontos ao redor da reta ajustada na correlação.

As equações anuais quando validadas na base mensal apresentaram melhores valores para o índice de ajustamento " $\mathrm{d}$ ", em vista das elaboradas para os demais agrupamentos de dados. Os modelos anuais que correlacionam as frações radiométricas ao coeficiente de transmissividade apresentaram melhor performance quanto ao $\mathrm{MBE}$, menor grau de espalhamento (RMSE) e melhor ajustamento (coeficiente " $d$ ") em relação às correlações com a razão de insolação.

Para $\mathrm{Kd} \times \mathrm{K}_{\mathrm{T}}$ os modelos sazonais apresentaram melhor desempenho nos meses de transição entre o período chuvoso e seco, e nos meses mais secos do ano, enquanto que na correlação $\mathrm{Kd} \times \mathrm{n} / \mathrm{N}$ observou-se comportamento contrário, com melhores estimativas para o período chuvoso. Ainda em $\mathrm{Kd} \times \mathrm{n} / \mathrm{N}$ as equações mensais obtiveram melhor performance para o período seco.

Em termos gerais, mediante a análise dos indicativos estatísticos, podese indicar que nos meses de seca apresentaram melhor estimativa com a 
utilização dos modelos mensais ou sazonais. De acordo com Recieri et al. (2002), a correlação polinomial de quarto grau não melhora o coeficiente de determinação em relação ao de $3^{\circ}$ grau e $2^{\circ}$ graus, no entanto, generaliza a estimativa da radiação para vários locais, não importando as condições climáticas, e podem projetar erros significativos com obtenção de valores fora dos intervalos ou da realidade de medidas.

De modo geral, os modelos anuais subestimam a radiação difusa medida, e as correlações entre as frações radiométricas e 0 coeficiente de transmissividade ( $\mathrm{Kd}$ e $\mathrm{Kd}^{\prime} \quad \mathrm{x} \quad \mathrm{K}_{\mathrm{T}}$ ) apresentam melhor desempenho para estimativa neste agrupamento.

Tabela 6 - Indicativos estatísticos obtidos para as equações geradas a partir das correlações de $\mathrm{Kd}$ com $\mathrm{K}_{\mathrm{T}}$ e $\mathrm{n} / \mathrm{N}$, na região de Sinop-MT

\begin{tabular}{|c|c|c|c|c|c|c|c|c|c|}
\hline \multirow[b]{3}{*}{ Período } & \multicolumn{7}{|c|}{$\mathrm{Kd} \times \mathrm{K}_{\mathrm{T}}$} & \multirow{2}{*}{\multicolumn{2}{|c|}{ Anual }} \\
\hline & \multicolumn{3}{|c|}{ Mensais } & \multicolumn{3}{|c|}{ Sazonais } & \multirow[b]{2}{*}{$\begin{array}{c}M B E \\
\left(\mathrm{MJ} \mathrm{m}^{-2}\right)\end{array}$} & & \\
\hline & $\begin{array}{c}\mathrm{MBE} \\
\left(\mathrm{MJ} \mathrm{m}^{-2}\right)\end{array}$ & $\begin{array}{l}\text { RMSE } \\
\left(M^{-2} \mathrm{~m}^{-2}\right)\end{array}$ & $d$ & $\begin{array}{c}\mathrm{MBE} \\
\left(\mathrm{MJ} \mathrm{m}^{-2}\right)\end{array}$ & $\begin{array}{l}\text { RMSE } \\
\left(M^{-2} \mathrm{~m}^{-2}\right)\end{array}$ & $d$ & & \multicolumn{2}{|c|}{ RMSE } \\
\hline Janeiro & $-0,67(2)$ & 1,75 (3) & 0,37 (3) & $-0,70(3)$ & $0,85(2)$ & $0,72(2)$ & $-0,48(1)$ & $0,65(1)$ & $0,83(1)$ \\
\hline Fevereiro & $-0,52(2)$ & $0,77(2)$ & $0,64(2)$ & $-0,67(3)$ & 0,94 (3) & 0,53 (3) & $-0,49(1)$ & $0,74(1)$ & $0,69(1)$ \\
\hline Março & $-0,44(3)$ & 0,94 (3) & $0,33(1)$ & $-0,19(1)$ & $0,92(1)$ & 0,18 (3) & $-0,36(2)$ & $0,93(2)$ & $0,29(2)$ \\
\hline Abril & $-0,24(1)$ & 0,66 (1) & $0,60(3)$ & $-0,36(2)$ & $0,73(2)$ & 0,65 (1) & $-0,48(3)$ & 0,78 (3) & $0,63(2)$ \\
\hline Maio & $-0,84(3)$ & 1,44 (1) & $0,73(1)$ & $-0,67(2)$ & $1,54(2)$ & $0,62(2)$ & $-0,65(1)$ & $1,57(3)$ & $0,58(3)$ \\
\hline Junho & $0,96(3)$ & 0,99 (2) & 0,69 (2) & $0,34(1)$ & 0,97 (1) & 0,71 (1) & $0,43(2)$ & 1,05 (3) & $0,64(3)$ \\
\hline Julho & $-0,04(2)$ & 0,87 (2) & $0,74(3)$ & 0,03 (1) & 0,85 (1) & $0,75(2)$ & 0,14 (3) & 0,91 (3) & $0,86(1)$ \\
\hline Agosto & $0,17(1)$ & $0,90(2)$ & $0,68(2)$ & $0,17(2)$ & 0,87 (1) & 0,70 (1) & $0,30(3)$ & 0,95 (3) & $0,63(3)$ \\
\hline Setembro & $-0,33(1)$ & 0,73 (1) & 0,58 (1) & $-0,41(2)$ & $0,81(2)$ & 0,45 (3) & $-0,58(3)$ & 0,89 (3) & $0,56(2)$ \\
\hline Outubro & $0,42(3)$ & 0,80 (3) & $0,62(2)$ & $0,26(2)$ & $0,63(2)$ & $0,61(3)$ & $0,08(1)$ & 0,59 (1) & $0,68(1)$ \\
\hline Novembro & $0,70(1)$ & 0,94 (1) & 0,51 (1) & $0,82(2)$ & $1,02(2)$ & $0,50(2)$ & $0,82(2)$ & 1,11 (3) & $0,42(3)$ \\
\hline Dezembro & $-0,33(1)$ & $1,25(1)$ & $0,37(2)$ & $-0,54(3)$ & $1,28(2)$ & 0,45 (1) & $-0,51(2)$ & $1,52(3)$ & $0,36(3)$ \\
\hline Verão & & & & $-0,41(2)$ & $0,82(2)$ & $0,55(2)$ & $-0,23(1)$ & 0,69 (1) & $0,67(1)$ \\
\hline Outono & & & & $-0,23(1)$ & 1,07 (1) & $0,82(1)$ & $-0,26(2)$ & $1,10(2)$ & $0,79(2)$ \\
\hline Inverno & & & & $0,08(1)$ & 0,94 (1) & $0,92(1)$ & $0,11(2)$ & $1,04(2)$ & 0,89 (2) \\
\hline Primavera & & & & $0,13(2)$ & 0,92 (1) & 0,47 (1) & 0,00 (1) & $1,11(2)$ & $0,37(2)$ \\
\hline \multirow[t]{2}{*}{ Anual } & & & & & & & $-0,15$ & 1,03 & 0,89 \\
\hline & \multicolumn{6}{|c|}{$\mathrm{Kd} \times \mathrm{n} / \mathrm{N}$} & & & \\
\hline Janeiro & $-0,48(3)$ & $1,36(3)$ & $0,67(2)$ & $-0,37(2)$ & $1,02(1)$ & $0,78(1)$ & $-0,32(1)$ & $1,02(2)$ & $0,78(1)$ \\
\hline Fevereiro & $0,14(1)$ & 1,35 (3) & 0,57 (1) & $-0,45(3)$ & $1,23(1)$ & $0,55(2)$ & $-0,44(2)$ & $1,25(2)$ & $0,55(2)$ \\
\hline Março & $-1,30(1)$ & $1,67(1)$ & $0,42(3)$ & $-1,51(3)$ & $1,79(3)$ & $0,40(2)$ & $-1,46(2)$ & $1,72(2)$ & $0,42(1)$ \\
\hline Abril & $-0,75(1)$ & $1,08(1)$ & 0,68 (1) & $-0,97(3)$ & $1,23(3)$ & $0,62(3)$ & $-0,88(2)$ & $1,13(2)$ & $0,64(2)$ \\
\hline Maio & $-0,97(3)$ & $1,83(3)$ & 0,47 (3) & $-0,83(2)$ & $1,72(2)$ & 0,59 (1) & $-0,63(1)$ & 1,67 (1) & $0,56(2)$ \\
\hline Junho & 0,45 (1) & 1,09 (1) & 0,77 (1) & $0,61(2)$ & $1,26(2)$ & $0,58(3)$ & 0,80 & $1,30(3)$ & $0,65(2)$ \\
\hline Julho & 0,09 (1) & $1,02(1)$ & $0,56(2)$ & $0,39(2)$ & 1,14 (3) & 0,50 (3) & $0,53(3)$ & $1,13(2)$ & $0,84(1)$ \\
\hline Agosto & $0,32(1)$ & $1,42(1)$ & $0,31(2)$ & $0,44(2)$ & $1,42(1)$ & $0,27(3)$ & 0,57 (3) & $1,49(2)$ & $0,32(1)$ \\
\hline Setembro & $-0,85(2)$ & $1,13(2)$ & $0,53(1)$ & $-0,81(1)$ & 1,09 (1) & $0,50(2)$ & $-1,28(3)$ & 1,49 (3) & $0,48(3)$ \\
\hline Outubro & $0,02(1)$ & $0,70(2)$ & $0,72(2)$ & $-0,16(2)$ & 0,58 (1) & 0,81 (1) & $-0,60(3)$ & $0,84(3)$ & $0,72(2)$ \\
\hline Novembro & 0,19 (2) & 0,99 (2) & $0,63(2)$ & $0,27(3)$ & 0,95 (1) & $0,66(1)$ & $-0,02(1)$ & $1,11(3)$ & 0,55 (3) \\
\hline Dezembro & $0,01(1)$ & $1,67(3)$ & $0,71(2)$ & $0,03(2)$ & 1,59 (1) & $0,72(1)$ & 0,07 (3) & $1,65(2)$ & $0,71(2)$ \\
\hline Verão & & & & $-0,43(1)$ & $1,07(1)$ & $0,66(1)$ & $-0,43(1)$ & $1,09(2)$ & $0,66(1)$ \\
\hline Outono & & & & $-0,56(2)$ & $1,28(2)$ & 0,78 (1) & $-0,42(1)$ & 1,25 (1) & $0,76(2)$ \\
\hline Inverno & & & & $-0,01(1)$ & $1,60(2)$ & $0,61(2)$ & $0,26(2)$ & $1,50(1)$ & 0,71 (1) \\
\hline Primavera & & & & $-0,02(1)$ & $1,13(1)$ & $0,76(1)$ & $-0,30(2)$ & $1,31(2)$ & $0,72(2)$ \\
\hline Anual & & & & & & & $-0,31$ & 1,33 & 0,65 \\
\hline
\end{tabular}

*Valores entre parênteses referem-se ao Vp 
Tabela 7 - Indicativos estatísticos obtidos para as equações geradas a partir das correlações de $\mathrm{Kd}^{\prime}$ com $\mathrm{K}_{\mathrm{T}}$ e $\mathrm{n} / \mathrm{N}$, na região de Sinop-MT

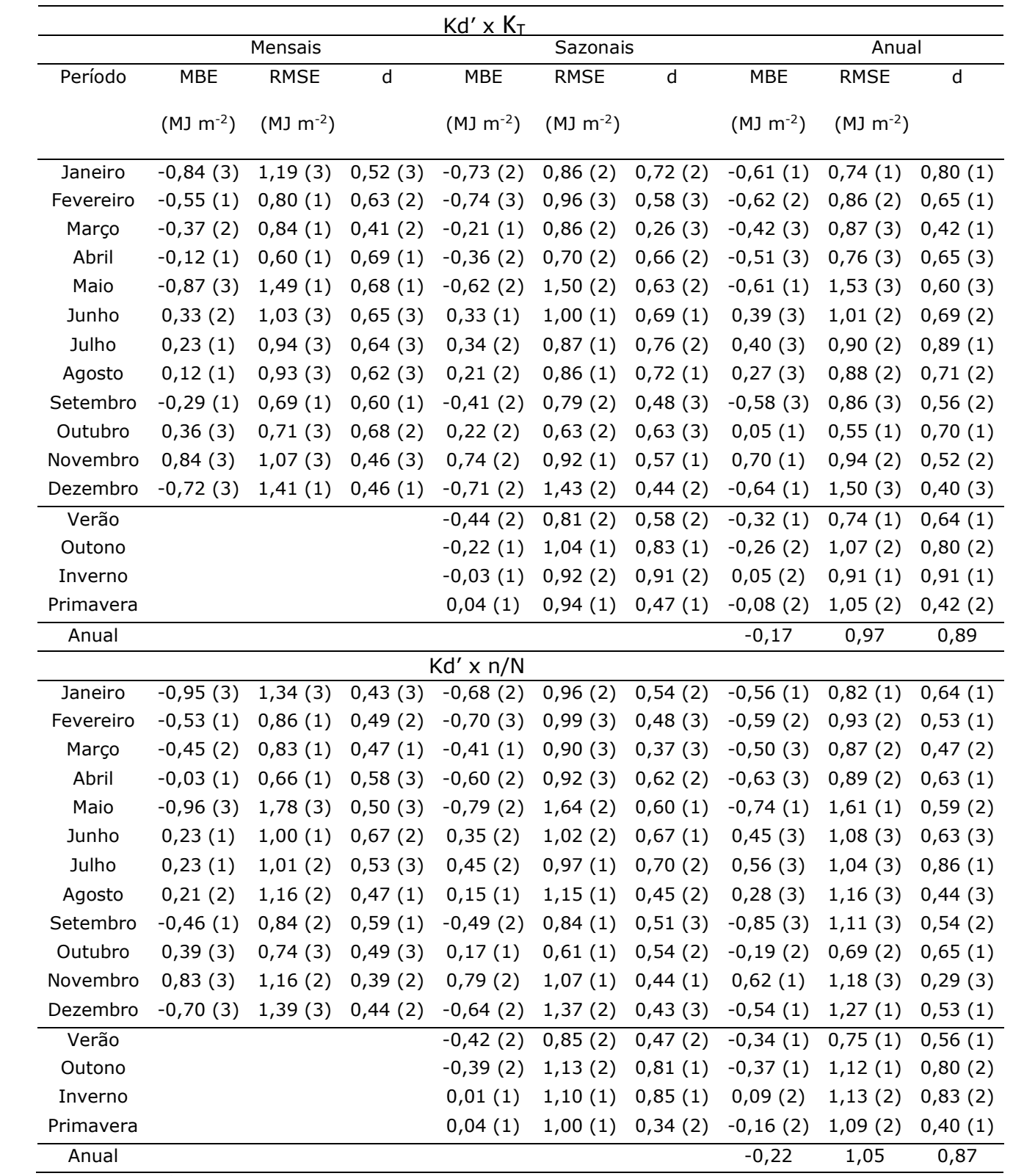

*Valores entre parênteses referem-se ao Vp

\section{CONCLUSÕES}

Os modelos estatísticos de estimativa da fração radiométrica $\mathrm{Kd}$ (difusa/global) baseados no coefieciente de transmissividade atmosférica $\left(\mathrm{K}_{\mathrm{T}}\right)$, distribuídos nos agrupamentos sazonais (estações do ano), geram boas estimativas da radiação difusa. 
As correlações $\mathrm{Kd}$ com a razão de insolação $(\mathrm{n} / \mathrm{N})$ mostraram-se mais fracas quando comparadas com $\mathrm{Kd} \times \mathrm{KT}$ e apresentam boas estimativas apenas na estação chuvosa por modelos sazonais.

\section{AGRADECIMENTOS}

À Fundação de Amparo à Pesquisa do Estado de Mato Grosso (FAPEMAT), pelo apoio financeiro concedido com bolsa de mestrado à aluna de pósgraduação.

\section{REFERÊNCIAS BIBLIOGRÁFICAS}

ALVARES, C. A.; STAPE, J. L.; Sentelhas, P. C.; GONÇALVES, J. L. DE M.; SPAROVEK, G. Köppen's climate classification map for Brazil. Meteorologische Zeitschrift, v.22, n.6, p.711-728, 2014.

BARBARO, S.; CANNATO, G.; COPPOLINA, S.; LEONE, C.; SINAGRA, E. Diffuse solar radiation statistics for Italy. Solar Energy, v.26, p.429-35, 1981.

BASHAHU, M. Statistical comparison of models for estimating the monthly average daily diffuse radiation at a subtropical African site. Solar Energy, v.75, p.43-51, 2003.

BERUSKI, G. C.; PEREIRA, A. B.; SENTELHAS, P. C. Desempenho de diferentes modelos de estimative da radiação solar global em Ponta Grossa, PR. Revista Brasileira de Meteorologia, v.30, n.2, p. 205-2013, 2015.

BOUKELIA, T. E.; MECIBAH, M-S.; MERICHE, I. E. General models for estimation of the monthly mean daily diffuse solar radiation (Case study: Algeria). Energy Conversion and Management, v.81, p.211-219, 2014.

BUTT, N.; NEW, M.; MALHI, Y.; COSTA, A. C. L. DA; OLIVEIRA, P.; SILVAESPEJO, J. E. Diffuse radiation and cloud fraction relationships in two contrasting Amazonian rainforest sites. Agricultural and Forest Meteorology, v.150, p.361368, 2010.

DAL PAI, A. Radiação solar difusa instantânea: avaliação dos fatores de correção anisotrópicos e modelos de estimativa. 2001. 81f. Dissertação (Mestrado em Agronomia-Energia na Agricultura), Faculdade de Ciências Agronômicas, Universidade Estadual Paulista, Botucatu, 2001.

DAL PAI, A. Anisotropia da irradiância solar difusa medida pelo método de sombreamento Melo-Escobedo: fatores de correção anisotrópicos e modelos de estimativa. 2005. 87f. Tese (Doutorado em Energia na Agricultura), Faculdade de Ciências Agronômicas, Unesp, Botucatu, 2005.

DAL PAI, A.; ESCOBEDO, J. F. Modelo de estimativa da radiação solar difusa instantânea. In: Simpósio em Energia na Agricultura, 1., 1999, Botucatu. Anais... Botucatu: UNESP, 1999. v.2, p.312-17.

DAL PAI, A.; ESCOBEDO, J. F.; CORREA, F. H. P. Numerical correction for the diffuse solar irradiance by the Melo-Escobedo shadowring measuring method. In: Ises Solar World Congress 2011, 2011, Kassel. 
DERVISHI, S.; MAHDAVI, A. Computing diffuse fraction of global horizontal solar radiation: A model comparison. Solar Energy, v.86, p.1796-1802, 2012.

DEVORE, J. L. Probabilidade e estatística: para engenharia e ciências. São Paulo, SP: Thomson Pioneira, 2006. 706p.

ELMINIR, H. K. Experimental and theoretical investigation of diffuse solar radiation: data and models quality tested for Egyptian sites. Energy, v.32, p.7382, 2007.

ELMINIR, H. K.; AZZAM, Y. A.; YOUNES, F. I. Prediction of hourly and daily diffuse fraction using neural network, as compared to linear regression models. Energy, v.32, p.1513-23, 2007.

ESCOBEDO, J. F.; DAL PAI, A.; OLIVEIRA, A. P.; SOARES, J. Radiações global, direta e difusa: ii - modelos de estimativa diários. In: Reunión Argentina, 10, y Latinoamericana de agrometeorologia, 4, 2004, Mar del Plata. Anais... Mar del Plata: AADA, 2004.

ESCOBEDO, J. F.; GOMES, E. N.; OLIVEIRA, A. P.; SOARES, J. Modeling hourly and daily fractions of UV, PAR and NIR to global solar radiation under various sky conditions at Botucatu, Brazil. Applied Energy, v.86, n.3, p.299-309, 2009.

GOMES, E. N.; ESCOBEDO, J. F. Modelos de estimativa da radiação fotossinteticamente ativa global e difusa em função da radiação de ondas curtas e do índice de claridade (KT). Irriga, v.7, n.2, p.122-129, 2002.

IQBAL, M. Correlation of average diffuse and beam radiation with hours of bright sunshine. Solar Energy, v.23, n.2, p.169-73, 1979.

IQBAL, M. An introduction to solar radiation. Canadá: Academic Press, 1983. $390 p$

JACOVIDES, C. P.; TYMVIOUS, F. S.; ASSIMAKOPOULOS, V. D.; KALTSOUNIDES, N. A. Comparative study of various correlations in estimating hourly diffuse fraction of global solar radiation. Renewable Energy, v.31, p.2492-504, 2006.

JAIN, P. C. A model for diffuse and global irradiation on horizontal surfaces. Solar Energy, v.45, p.301-8, 1990.

JIANG, Y. Estimation of monthly mean daily diffuse radiation in China. Applied Energy, v.86, p.1458-64, 2009.

KUMAR, R.; UMANAND, L. Estimation of global radiation using clearness index model for sizing photovoltaic system. Renewable Energy, v.30, n.15, p.222133, 2005.

KOUSSA, M.; MALEK, A.; HADDADI, M. Statistical comparison of monthly mean hourly and daily diffuse and global solar irradiation models and a Simulink program development for various Algerian climates. Energy Conversion and Management, v.50, n.5, p.1227-1235, 2009.

KUO, C-W.; CHANG, W-C.; CHANG, K-C. Distribution of solar diffuse fraction in Taiwan. Energy Procedia, v.57, p.1120-1129, 2014.

LI, H.; MA, W.; WANG, X.; LIAN, Y. Estimating monthly average daily diffuse solar radiation with multiple predictors: A case study. Renewable Energy, v.36, p.1944-1948, 2011. 
LIU, R. Y. H.; JORDAN, R. C. The interrelationship and characteristics distribution of direct, diffuse and total solar radiation. Solar Energy, v.4, n.3, p.1-19, 1960.

MELO, J. M. D. Desenvolvimento de um sistema para medir simultaneamente radiações global, difusa e direta. 1994. 130 f. Tese (Doutorado em Agronomia Energia na Agricultura), Faculdade de Ciências Agronômicas, Universidade Estadual Paulista, Botucatu, 1994.

MUNEER, T., MUNAWWAR, S. Potential for improvement in estimation of solar diffuse radiation. Energy Conversion and Management, v.47, p.68-86, 2006.

OliveirA, A. P., ESCOBEDO, J. F., MACHADO, A. J., SOARES, J. Correlation models of diffuse solar radiation applied to the city of Sao Paulo, Brazil. Applied Energy, v.71, n.1, p.59-73, 2002.

ORGILL, J. F.; HOLLANDS, K. G. T. Correlations equation for hourly diffuse radiation on a horizontal surface. Solar Energy, v.19, p.357-9, 1977.

RECIERI, R. P.; KOEHLER, K.; KOEHLER, A. C.; SOUZA, S. N. M. de. Modelos de estimativa da radiação difusa diária em Cascavel. In: Encontro de Energia no Meio Rural, 4., 2002, Campinas. Anais... Campinas: AGRENER, 2002.

REINDLE, D. T.; BECKMAN, W. A.; DIFFIE, J. A. Diffuse fraction correlations. Solar Energy, v.45, p.1-7, 1990.

SETTE, D. M.; TARIFA, J. R. A estrutura pluvial e as paisagens no Mato Grosso Brasil. In: Simpósio Brasileiro de Climatologia Geográfica, 4, Rio de Janeiro, Rio de Janeiro. Anais... Rio de Janeiro: UFRJ, 2000.

SOUZA, A. P. DE; ESCOBEDO, J. F.; DAL PAI, A.; GOMES, E. N. Estimativas das componentes da radiação solar incidente em superfícies inclinadas baseadas na radiação global horizontal. Revista Brasileira de Engenharia Agrícola e Ambiental, v.15, n.3, p.277-288, 2011.

SOUZA, A. P. DE; ESCOBEDO, J. F. Estimativas das radiações direta e difusa em superfícies inclinadas com base na razão de insolação. Revista Brasileira de Ciências Agrárias, v.8, n.3, p.492-502, 2013.

SOUZA, A. P.; MOTA, L. L.; ZAMADEI, T.; MARTIM, C. C.; ALMEIDA, F. T.; PAULINO, J. Classificação climática e balanço hídrico climatológico no Estado de Mato Grosso. Nativa, v.1, n.1, p.34-43, 2013.

SOUZA, A. P.; ZAMADEI, T.; MONTEIRO, E. B.; CASAVECCHIA, B. H. Transmissividade atmosférica da radiação global na região Amazônica de Mato Grosso. Revista Brasileira de Meteorologia, v.31, n.4, p.639-648, 2016

STONE, R. J. Improved statistical procedure for the evaluation of solar radiation estimation models. Solar Energy, v.51, n.4, p.289-291, 1993.

TANAKA, A. A.; SOUZA, A. P.; KLAR, A. E.; SILVA, A. C.; GOMES, A. W. A. Evapotranspiração de referência estimada por modelos simplificados para o Estado de Mato Grosso. Pesquisa Agropecuária Brasileira, v.51, n.2, p.91-104, 2016.

TEKE, A.; YILDIRIM, H. B.; ÇELIK, Ö. Evalution and performance comparison of different models for the estimation of solar radiation. Renewable and Sustainable Energy Reviews, v.50, p.1097-1107, 2015. 
TASDEMIROGLU, E.; SEVER, R. Estimation of monthly average daily, horizontal diffuse radiation in Turkey. Energy, v.16, n.4, p.787-90, 1991.

ULGEN, K.; HEPBASLI, A. Diffuse solar radiation estimation models for Turkey's big cities. Energy Conversion and Management, v.50, p.149-56, 2009.

WILLMOTT, C. J. On the validation of models. Physical Geography, v.2, n.2, p.184-194, 1981. 